\title{
RDUS
}

Revue de DROIT

UNIVERSITÉ DE SHERBROOKE

Titre :

Auteur(s): $\quad$ Lionel ZEVOUNOU

Revue : $\quad$ RDUS, 2016, volume 46, numéro 1

Pages: $\quad 205-246$

ISSN :

Éditeur : $\quad$ Université de Sherbrooke. Faculté de droit.

URI : $\quad$ http://hdl.handle.net/11143/11320

DOI : $\quad$ https://doi.org/10.17118/11143/11320

0317-9656
(RE) PENSER LE DROIT ADMINISTRATIF AVEC JACQUES CAILLOSSE 
Page vide laissée intentionnellement. 


\section{(RE) PENSER LE DROIT ADMINISTRATIF AVEC JACQUES CAILLOSSE}

\section{par Lionel ZEVOUNOU*}

Au Canada comme en Europe, l'État connaît de profonds bouleversements. La France n'est guère épargnée par ce mouvement. Peut-être plus que les autres pays de l'Union européenne, l'interventionnisme traditionnel de l'administration française est soumis à des politiques publiques d'inspiration néolibérale; d'un autre côté, le dogme de l'État unitaire est de plus en plus ébranlé par un ensemble de réformes décidées au niveau de l'Union européenne. Le droit administratif français est, depuis plusieurs années, traversé par ces différentes tensions. Cet article propose d'introduire le lecteur à la pensée de Jacques Caillosse à travers son dernier ouvrage, L'État du droit administratif. Cette pensée d'inspiration critique permet de saisir les enjeux politiques qui traversent le droit et l'action publique contemporains en France**.

In Europe as well as in Canada, governments are undergoing major changes. France is no exception. Possibly even more than in other countries of the European Union, the traditional interventions of the French government are subject to influence of neo-liberal politics. On the other hand, the doctrine of a unitary state has been increasingly weakened due to various reforms adopted by the European Union. For many years, French public law has also been affected by these various tensions. This article proposes to present the thought of Jacques Caillosse, as presented in his latest book, L'État du droit administratif. His critical study enables one to better understand the political issues at stake in contemporary French public law and public action.

* $\quad$ Maître de conférences en droit public, Université Paris Ouest Nanterre La Défense, membre du Centre de Recherche en Droit Public (CRDP EA 381) et membre du CODESRIA (Conseil pour le développement et la recherche en sciences sociales en Afrique).

${ }^{* *}$. Je remercie chaleureusement le comité de rédaction ainsi que les évaluateurs de la présente revue. Je remercie par la même occasion deux collègues et amis qui se reconnaîtront pour leurs soutiens respectifs lorsqu'il s'est agi de publier ce travail. 


\section{SOMMAIRE}

I- Ce que penser le droit administratif veut dire ......... 217

II- Une réflexion pluridisciplinaire de juriste ............. 226

III- Un regret et deux critiques ............................ 236 
Selon qu'elle est employée au Canada ou en France, l'expression "droit administratif " ne se réfère pas toujours aux mêmes objets. Que l'on sache, c'est au début des années 1960 que le droit administratif apparaît dans le champ académique québécois' ${ }^{1}$. Si l'on en croit l'avant-propos de Barbe à l'ouvrage Droit administratif canadien et québécois, il s'agit d'

(...) une étape dans l'évolution de la doctrine relative à notre droit administratif. Il [l'ouvrage] comble un vide dans la doctrine d'expression française. En faisant cette synthèse, ce livre permettra une évolution plus ordonnée de ce domaine du droit encore peu exploré, mais qui prend sans cesse de l'importance à cause de l'intervention de plus en plus considérable de l'État ${ }^{2}$.

On peut se risquer à dire que ce point de vue défendu par Barbe est largement inspiré de la culture juridique française de droit administratif. Il n'est qu'à lire le plan suivi3, les parcours académiques des divers contributeurs ou, mieux encore, la définition proposée par Rambourg du droit administratif, inspirée par De Laubadère :

Le droit administratif est cette branche du droit public interne qui comprend l'organisation et l'activité de ce qu'on appelle couramment l'administration, c'est-à-dire l'ensemble des autorités, agents et organismes chargés, sous l'impulsion des pouvoirs politiques, d'assurer les

1. M. LALONDE, Cours de droit administratif approfondi: le contrôle de l'administration par le contentieux judiciaire, Ottawa, Faculté de droit de l'Université d'Ottawa, 1960-1961; Patrice GARANT, Notes du cours de droit public, Québec, Faculté de droit de 1'Université Laval, 1972. Nous tirons cette affirmation de la démonstration de René Dussault : René DussaulT, Traité de droit administratif canadien et québécois, t.1, Québec, Les Presses de 1'Université Laval, 1974, p. 21-22.

2. Raoul-P. BARBE (dir.), Droit administratif canadien et québécois, vol. 4, Ottawa, Université d'Ottawa, 1969, à la page 7.

3. Le plan s'inspire fort largement de celui d'André de Laubadère : André DE LAUBADĖRE, Traité de droit administratif, 6e éd., t. 1 et 2, Paris, L.G.D.J., 1973. 
multiples interventions des pouvoirs politiques, d'assurer les multiples interventions de l'État moderne ${ }^{4}$.

En extrapolant encore, les références à Weil, Rivero, Eisemann $^{5}$ ou Francis-Paul Benoit pourraient laisser accroire que les disciplines de droit administratif canadien et français s'attachent aux mêmes problématiques.

Ne nous y trompons pas : le droit administratif canadien, québécois en particulier, prend racine dans un système de common law. Certes, l'anathème de Dicey à l'encontre des systèmes juridiques latins ou continentaux est aujourd'hui largement derrière nous ${ }^{6}$. Plus personne ne soutient sérieusement que la soumission de l'administration à un juge de droit commun serait plus protectrice du justiciable que celle à un juge administratif spécial enclin à faire la part belle à l'exorbitance de l'administration ${ }^{7}$. Les avatars de l'histoire expliquent la prégnance du modèle anglais sur le droit administratif québécois. Le préambule de l'Acte de l'Amérique du Nord britannique de 1763 impose en effet "(...) qu'à moins de dispositions spécifiques contraires, les principes généraux du droit constitutionnel et du droit administratif copieront ceux du Royaume-Uni " 8 . Ce constat

4. Michel RAmBOURG, "Notions générales sur le droit administratif ", dans Raoul-P. BARBE (dir.), Droit administratif canadien et québécois, Ottawa, Université d'Ottawa, 1969, à la page 11.

5. R. DusSAUlT, préc., note 1, p. 3-9.

6. On oserait même dire que la critique de Dicey a largement été exagérée dans la mesure où de nombreux juristes français ont eux aussi pris part à la critique de la justice administrative. Par exemple : Nobert FOULQUIER (prés.), "Une conception d'ensemble du droit administratif. René Jacquelin, Paris, V. Giard \& E. Brière, 1899 ", (2006) R.F.D.A. 527; Grégoire Bigot, "H. Berthélémy ou la tradition du libéral-étatisme ", dans Nader HaKim et Fabrice MElleray (dir.), Le Renouveau de la doctrine française. Les grands auteurs de la pensée juridique au tournant $d u X X e ̀$ siècle, Paris, Dalloz, 2009, aux pages 199-213.

7. Jean-Bernard AUBY et Mark FREEDLAND (dir.), La distinction droit public, droit privé : regards croisés français et britanniques, Paris, PanthéonAssas, 2004.

8. R.-P. BARBE (dir.), préc., note 2, à la page 30. 
n'autorise pas une vision hégémonique : le droit paroissial constitue à maints égards une spécificité, certes minime, du système québécois9. Telle qu'elle ressort de la doctrine, la spécificité du droit administratif québécois renvoie en définitive davantage aux dispositions du Code civil du Bas-Canada de 1866 qu'à l'existence d'une juridiction administrative proprement dite ${ }^{10}$. La question de l'originalité du droit administratif québécois s'est d'ailleurs posée à cet égard. Le juge Archambault de la Cour supérieure du Québec déclarant que "l'on ne doit pas rechercher dans les auteurs français et la jurisprudence française les principes de notre loi administrative publique, car celle-ci a été tirée entièrement du droit anglais "11. Les choses sont, à l'analyse, plus complexes puisque l'administration est à la fois soumise au droit commun de la common law, au droit civil du Code de 1994 et

9. Le droit paroissial s'entend de cette branche du droit qui régit le droit temporel des paroisses. Ses sources sont nombreuses et éparses. On trouve parmi elles plusieurs décisions d'intendants, du Conseil Souverain et du Conseil d'État du Roi. L'expression "droit administratif " employée s'agissant du droit paroissial s'apparente plutôt à un avatar du droit public tel que pouvait l'entendre le droit romain (on renvoie sur ce point à Georges CHEVRIER, "Remarques sur l'introduction et les vicissitudes de la distinction "jus privatum" et "jus publicum" dans les œuvres des anciens juristes français ", (1952) A.D.P. 5, 5-77. Sur cette question : Hector L. LANGEvin, Droit administratif ou manuel des paroisses et fabriques, 2e éd., Québec, Imprimerie du Canadien, 1878; JeanFrançois Pouliot, Le droit paroissial de la province du Québec, Fraserville, Imprimerie le Saint-Laurent, 1919.

10. Le système français se caractérise par l'institution de deux cours suprêmes : la Cour de cassation (ci-après Ccass) d'un côté pour ce qui concerne les litiges de droit privé, le Conseil d'État (ci-après $\mathrm{CE}$ ) de l'autre pour ce qui concerne les litiges liés à l'administration. On parle usuellement de dualisme juridictionnel pour rendre compte de ce système. Chacun des deux systèmes comprend des juges du fond: Tribunaux administratifs, Cours administratives d'appel pour l'ordre administratif, Tribunaux d'instance, Tribunaux de grande instance, Cours d'appel pour l'ordre judiciaire. Rappelons que cette présentation n'est que synthétique. Elle ne tient pas compte des juridictions spécialisées présentes dans les deux ordres juridictionnels.

11. R. Dussault, préc., note 5, p. 18. 
à un ensemble de législations spéciales ${ }^{12}$. De ce point de vue, le droit administratif canadien n'est pas différent d'un système de Rule of Law $^{13}$ : si l'administration n'a pas de juge spécifique ${ }^{14}$, elle est en revanche soumise à des règles pour partie dérogatoires du droit commun. Sans doute ce système explique-t-il l'éclectisme de la recherche canadienne dans le champ académique. Les thèmes abordés par la doctrine portent autant sur le contrôle du juge ${ }^{15}$ que sur la compétence des autorités administratives, la mondialisation ${ }^{16}$, le droit des autochtones ou le droit des nouvelles technologies ${ }^{17}$.

La formation du droit administratif français est toute différente. Historiquement, le Conseil d'État a d'abord été conseiller du gouvernement avant d'être juge18. Cette proximité a d'ailleurs alimenté un fort soupçon de partialité à l'encontre du juge administratif ${ }^{19}$. Le statut de la magistrature administrative

12. Pour une discussion de ce point: Pierre Lemieux, "Le Code civil du Québec et l'administration publique ", (2008) 1 Acta Universitatis Lucian Blaga 56, 56-66.

13. On renverra sur ce point à : Richard A. Cosgrove, The Rule of Law: Albert Venn Dicey, a Victorian Jurist, London, Macmillan, 1980.

14. Entendu de juge de droit commun spécifique, ce qui ne signifie pas que certains contentieux puissent relever de juridictions spéciales voire de juridictions plus générales, telles que la Cour fédérale du Canada. Sur cette question: Geneviève CARTIER et Suzanne ComTois, "La reconnaissance d'une forme mitigée de dualité de juridictions en droit administratif canadien ", (1995) 47 Revue Internationale de Droit Comparé 51, 51-76.

15. Par exemple, Suzanne Comtois, Paul Daly, Ian Demers, Dominique Guimon, Nicolas LAMBERT et Mario NormAndin, Contrôle judiciaire, Montréal, Lexis-Nexis Canada, 2014.

16. Daniel Mockle, La gouvernance, le droit, l'État : la question du droit dans la gouvernance publique, v. 11, coll. "Mondialisation et droit international ", Bruxelles, Bruylant, 2008.

17. Nicolas W. VermeYs, Droit codifié et nouvelles technologies : le Code civil, Montréal, Éditions Yvon Blais, 2015.

18. François BuRDEAU, Histoire du droit administratif, coll. "Droit public ", Paris, P.U.F., Éditions Thémis, 1995.

19. Par exemple : Charles EISENMANn, Un dogme faux : l'autonomie du droit administratif, Madrid, Instituto de estudios de administración local, 1969, p. 419-438; Roland DRAGO et Marie-Anne FRISON-RochE, "Mystères 
n'est prévu par aucun texte de la Constitution de la Ve République ${ }^{20}$. À la fois juge et conseiller du gouvernement, il a longtemps été critiqué pour son manque de sensibilité à l'égard des droits du justiciable ${ }^{21}$. La jurisprudence du Conseil d'État reste essentiellement prétorienne : à l'inverse du droit civil, elle ne figure pas dans un code, même s'il convient de prendre acte du mouvement de codification du droit administratif mis en oeuvre par des commissions elles-mêmes présidées par des conseillers d'État22.

Le modèle d'État unitaire à la française est de plus en plus concurrencé par un fédéralisme européen qui ne dit pas son nom $^{23}$. Cette concurrence s'exerce tant sur le terrain du droit que

et mirages des dualités des ordres de juridiction et justice administrative ", (1997) 41 A.P.D. 135, 135-148.

20. Sur la revendication d'un statut clair de la magistrature administrative sur le modèle du juge judiciaire, voir la tribune : ConseIL SyNDICAL DU SYNDICAT DE LA JUSTICE ADMINISTRATIVE, " Halte au procès d'intention ", AJDA, 2016, p. 809.

21. Jean Rivero, "Nouveaux propos naïfs d'un Huron sur le contentieux administratif ", (1979) 31 E.D.C.E. 27, 27-30.

22. Jean-Marc SAUVÉ, "Les 25 ans de la relance de la codification ", discours prononcé le 13 octobre 2015 à l'Institut Français des Sciences Administratives (IFSA), en ligne : http://www.conseil-etat.fr/content/ download/48522/423945/version/ 1/file/2015-10-13\%20-\%20Colloque \%20pour\%20les\%2025\%20ans\%20de\%20la\%20relance\%20de\%20la\% 20codification\%20IFSA.pdf> (consulté le 21 avril 2016); Georges VEDEL, "Le droit administratif peut-il être indéfiniment jurisprudentiel? ", (1979) 31 E.D.C.E. 31; Gilles J. GuGLIELMI, "L'idée de codification dans la construction du droit administratif français au XIXe siècle ", (1996) 8 Annuaire d'histoire administrative européenne 109, 109-133; Fabrice MELLERAY, "Le droit administratif doit-il redevenir jurisprudentiel? Remarques sur le déclin paradoxal de son caractère jurisprudentiel ", (2005) 12 A.J.D.A. 637; Pascale Gonod et Olivier JouANJAN, " À propos des sources du droit administratif, brèves notations sur de récentes remarques ", (2005) 18 A.J.D.A. 992.

23. Dernier épisode juridique en date, l'avis défavorable du Conseil d'État sur l'introduction de la Charte des langues régionales : Cons. d'Ét. Ass., 30 juillet 2015, Projet de loi constitutionnelle autorisant la ratification de la Charte des langues régionales ou minoritaires, Rec. Cons. d'Ét., $n^{\circ} 390.268$. On peut se référer à cet égard à la livraison de la revue 
sur celui des politiques publiques. Depuis plusieurs années, l'office du juge administratif s'exerce dans le cadre d'un État unitaire et d'une administration en plein bouleversement sous l'influence de l'Union européenne et de la Convention européenne des droits de l'homme. Sur de nombreux aspects, son office s'est rapproché, au fil des jurisprudences, du droit administratif canadien. Les deux ordres juridictionnels partagent en effet une politique jurisprudentielle commune, recherchant l'équilibre délicat entre l'efficacité administrative et la protection des droits du justiciable. Comme l'a souligné la Cour suprême du Canada sur le sujet dans l'arrêt Dunsmuir c. Nouveau-Brunswick de 2008 :

Le caractère raisonnable tient principalement à la justification de la décision, à la transparence et à l'intelligibilité du processus décisionnel, ainsi qu'à l'appartenance de la décision aux issues possibles acceptables pouvant se justifier au regard des faits et du droit $^{24}$.

Si les objectifs de politique jurisprudentielle convergent, les moyens pour y parvenir diffèrent. Le juge canadien fait en effet application du principe de déférence - sous réserve que la décision prise par l'administration soit "déraisonnable ", principe fondé sur la reconnaissance d'un pouvoir discrétionnaire de l'administration reconnu sous certaines conditions: l'expertise et la reconnaissance d'un pouvoir discrétionnaire attribué à l'administration 25 . Aussi, à la différence du droit français, le juge

Pouvoirs locaux de septembre 2013: InstiTUT DE LA GOUVERNANCE TERRITORIALE ET DE LA DÉCENTRALISATION, " La territorialisation du droit. Quelle relation entre la norme et l'espace? ", (2013), 98 Pouvoirs locaux 39, 39-110.

24. $\quad$ Dunsmuir c. Nouveau-Brunswick, 2008 CSC 9, par. 47.

25. Geneviève CARTIER, "Le principe de précaution et la déférence judiciaire en droit administratif ", (2002) 43 C. de D. 79, 79-101. 
ne peut-il pas substituer son appréciation à celle de l'administration ${ }^{26}$.

Sans entrer dans des développements trop complexes, évoquons deux points significatifs. La banalisation progressive de l'intervention administrative sur le modèle de l'entreprise, d'une part, et la protection accrue du justiciable, d'autre part. La politique jurisprudentielle du Conseil d'État s'est, à cet égard, sensiblement ouverte aux raisonnements et aux méthodes proposés par les cours de Luxembourg et de Strasbourg. S'y ajoute depuis quelques années l'extension du contrôle sur le droit souple 27 et l'introduction de la "question prioritaire de constitutionnalité " (ci-après : "QPC"), qui a accéléré la convergence des politiques jurisprudentielles entre le Conseil d'État, la Cour de cassation et le Conseil constitutionnel28. En dépit de ces changements, abondamment soulignés par la

26. Conclusions de Madame Claire Legras, sous l'arrêt Société ATOM : Cons. d’Ét. Ass., 16 février 2009, Société ATOM, R.F.D.A. 2009. 268.

27. Sur cette question: Benjamin LAVERGNE, "Droit souple " dans Michel Bazex, Gabriel Eckert, Régis Lanneau, Christophe Le Berre, Bertrand Du MARAIS et Arnaud SÉE (dir.), Dictionnaire des régulations, Paris, LexisNexis, 2016, aux pages 598-606; CONSEIL D’ÉTAT, Le droit souple, coll. "Études et documents ", CONSEIL D'ÉTAT, Paris, La documentation française, 2013. Plus récemment deux arrêts étendent le contrôle du juge sur le droit souple des autorités administratives indépendantes: Cons. d'Ét. Ass., 21 mars 2016, Société NC Numericable, Rec. Cons. d'Ét., n³900023 et Cons. d'Ét. Ass., 21 mars 2016, Société Fairvesta International GMBH et autres, Rec. Cons. d'Ét., n³68082, 368084.

28. Introduite par une réforme constitutionnelle de 23 juillet 2008, la question prioritaire de constitutionnalité se définit comme le "droit reconnu à toute personne qui est partie à un procès ou une instance de soutenir qu'une disposition législative porte atteinte aux droits et libertés que la Constitution garantit. Si les conditions de recevabilité sont réunies, il appartient au Conseil constitutionnel, saisi sur renvoi par le Conseil d'État et la Cour de cassation de se prononcer et, le cas échéant, d'abroger la disposition législative ", en ligne : <http://www.conseilconstitutionnel.fr/conseil-constitutionnel/francais/la-questionprioritaire-de-constitutionnalite/decouvrir-la-qpc/decouvrir-la-questionprioritaire-de-constitutionnalite-qpc.47106.html> (consulté le 14 avril 2016). 
doctrine ${ }^{29}$, un certain nombre de particularismes demeurent. Au premier rang de ceux-ci : la place centrale occupée par le juge administratif dans l'espace académique français. Ce sont ces particularismes qui permettent de se figurer les questions traversant aujourd'hui la doctrine de droit administratif en France.

On ne reviendra pas, sauf pour en prendre acte, sur la prépondérance de la haute administration française, tant dans le fonctionnement de l'État que de son droit ${ }^{30}$. Pour en prendre la mesure, il suffit de rappeler la place du Conseil d'État dans le droit public en général et dans le droit administratif en particulier ${ }^{31}$. Le jury du concours d'agrégation, qui désigne, pour l'ensemble des facultés de droit, les professeurs de droit public, comporte toujours un membre du Conseil d'État; les nouveaux professeurs ont d'ailleurs parfois le privilège d'être conviés par le vice-président du Conseil d'État, cette rencontre étant l'occasion pour l'institution d'exposer sa vision sur les rapports avec la doctrine ${ }^{32}$. En siégeant dans différents comités de revues et par le moyen d'articles, de contributions, de publications de conclusions

29. Pour un exemple parmi de multiples : Yves GaUdEMET, "L'avenir de la juridiction administrative ", (1979) Gaz. Pal. 511, 511-519; Yves GAUDEMET, "Monde économique et justice administrative : la mesure d'une critique ", (1995) 1 Justices 52, 52-62; Roland DRAGRO, Un nouveau juge administratif (Écrits en hommage à J. Foyer), Paris, P.U.F., 1997, p. 451-462.

30. Grégoire Bigot, Ce droit que l'on dit administratif... Études d'histoire du droit public, Paris, La Mémoire du Droit, 2015, p. 87-118; Jacques CHEVAllier, "L'élite politico-administrative: une interpénétration discutée ", (1997) 80 Pouvoirs 89, 89-100.

31. Par exemple : Rapport public 2006 du Conseil d'État, Sécurité juridique et complexité du droit, Paris, La documentation française, 2006 et Cons. d'Ét. Ass., 24 mars 2006, Société KPMG et Société Ernst et Young et autres, G.A.J.A. 2011. 874-887 (conclusions Y. Aguila, R.F.D.A. 2006. 463).

32. Intervention du 12 septembre 2014 lors du petit déjeuner donné en l'honneur de la promotion 2013-2014 des agrégés de droit public, en ligne : <http://www.conseiletat.fr/content/download/33657/290790/ version/1/file/promotiondesagregesdedroitpublic2013_2014_ 230892014.pdf> (consulté le 16 décembre 2015). 
de rapporteurs publics, les membres du Conseil d'État participent activement à la production doctrinale française: il existe d'ailleurs, depuis février 2015, un prix de thèse du Conseil d'État. Le rapport annuel de l'institution initie très clairement un agenda doctrinal $^{33}$. Le statut de professeur associé des membres du Conseil leur permet de vulgariser une certaine vision du droit administratif, celle du Conseil d'État en l'occurrence ${ }^{34}$. Faut-il ajouter que les juges français à la Cour de justice de l'Union européenne et à la Cour européenne des droits de l'homme proviennent pour certains... du Conseil d'État.

Ce rapide panorama donne une idée de la position centrale qu'occupe l'institution dans le champ du droit public français. Il est à peine besoin de souligner dans ces conditions que le droit administratif tel qu'il est généralement exposé dans les facultés de droit se réfère au droit de la jurisprudence administrative ${ }^{35}$. La situation est en réalité plus complexe, dans la mesure où les solutions du juge administratif sont souvent critiquées par la doctrine; rares sont ceux, en revanche, qui se hasardent à une critique, à la fois desdites solutions, mais aussi de la manière dont fonctionne plus largement le champ disciplinaire du droit administratif. Jacques Caillosse est de ceux-là. Sa réflexion sur le droit administratif ramène à l'urgence d'en repenser la discipline d'un point de vue théorique, dans un contexte que traversent la plupart des États occidentaux: transformation de l'État sous l'impulsion de politiques néolibérales, crise de la représentation politique, complexité croissante des institutions et des normes applicables à l'administration.

33. Un exemple parmi d'autres : Raymond ODEnT, Contentieux administratif, t.1, Paris, Dalloz, 2007, p. 7-11 (préface R. Denoix de Saint Marc).

34. Sur la question du statut des personnels associés dans les universités françaises: Olivier BEAUD, "Les facultés de droit dans la réforme universitaire. Les personnels associés à l'université : une singulière discordance entre un statut privilégié et le mode d'accès à ce statut ", (2009) 4 R.D.P. 959, 959-991.

35. Sur cette question: Jacques CAILlosse et Olivier Renaudie, Le Conseil d'État et l'Université, coll. "Thèmes et commentaires ", Paris, Dalloz, 2015. 
Sa pensée, laquelle nous proposons ici de mettre en perspective, permet de saisir les transformations profondes que connait depuis plusieurs années le droit administratif français. Ces transformations, Jacques Caillosse les appréhende à partir de référents culturels communs à la culture juridique nordaméricaine : on pense ici aux Critical Legal Studies ${ }^{36}$. Ancré dans une culture de sciences sociales, son propos constitue l'un des meilleurs points d'entrée pour qui cherche à comprendre les évolutions du droit administratif français contemporain. L'urgence d'appréhender le droit administratif d'un point de vue critique se justifie dans un contexte où le monopole des facultés de droit est, depuis un certain temps, profondément remis en cause. Sur le modèle nord-américain, l'école de droit de Sciences $\mathrm{Po}^{37}$ se pose en alternative d'une certaine tradition d'enseignement du droit: le droit des manuels s'opposant alors au droit des praticiens. Cette concurrence entre école et faculté de droit s'exerce pourtant à armes inégales : là où Sciences Po bénéficie de fonds publics et privés, l'Université peine à trouver des financements publics adéquats. Ainsi assiste-t-on à la transformation profonde, au-delà $\mathrm{du}$ droit administratif, du système français de l'enseignement

36. Sur la réception de ce courant dans la doctrine canadienne, voir : Patrick MAcklem, "Of Texts and Democratic Narratives (Review Essay of Hutchinson, Dwelling on the Threshold) ", (1991) 41 U.T.L.J. 114, 114145.

37. Précisons que le système de l'enseignement supérieur français reste fragmenté entre les grandes écoles, les universités et les instituts de recherche (CNRS, EHSS, IRD). Les grandes écoles accueillent historiquement l'élite républicaine destinée à la haute administration ou à l'entreprise. Pendant longtemps, les facultés ont gardé le monopole de l'enseignement du droit. En autorisant la délivrance des diplômes de droit par l'IEP de Paris (Sciences Po), un arrêté du 21 mars 2007 met fin à ce monopole. Cet arrêté a été jugé valide par le Conseil d'État : Cons. d'État, 28 juillet 2008, Syndicat autonome du personnel enseignant des sciences juridiques, politiques, économiques et de gestion des universités et Société des Professeurs des facultés de droit, Rec. Cons. d'Ét., $\mathrm{n}^{\circ} 306321$. Sur cette question de manière générale : Agnès VAN ZANTEN, "L'ouverture sociale des grandes écoles: diversification des élites ou renouveau des politiques publiques d'éducation?", (2010) 79 Sociétés contemporaines 69, 69-95. 
supérieur. Ce processus de transformation touche autant l'institution des facultés de droit que le droit lui-même. Sur ce dernier point, le droit administratif global apparaît comme un sérieux concurrent du droit administratif français. Autant de problèmes auxquels la pensée de Jacques Caillosse apporte des clés de lecture.

\section{I- Ce que penser le droit administratif veut dire}

Chacun sait la place singulière que Jacques Caillosse, professeur émérite de droit public à l'université Paris II PanthéonAssas, occupe au sein du droit administratif. Plus et mieux que nul autre, il ne se contente pas de décrire le droit administratif mais s'efforce, depuis de nombreuses années, d'en penser ses évolutions et ses modes si particuliers de production, ses influences et ses limites. Le regard qu'il porte sur le discours doctrinal est, à cet égard, loin d'être irénique. Il n'existe pas pour l'auteur un seul chemin pour comprendre le droit administratif. Son épistémologie se veut ouverte tant sur l'objet qu'est le droit administratif que sur la manière de l'appréhender, y compris par le recours aux sciences sociales.

Par les récents travaux consacrés au mouvement "critique du droit " ${ }^{38}$, on sait la solide culture pluridisciplinaire que Jacques Caillosse a acquise au regard du brassage entretenu par le Centre universitaire de recherches administratives et politiques de Picardie (CURRAP). Ce centre s'est distingué par son positionnement critique, tant sur le droit que sur l'action administrative. Voilà qui donne déjà un indice sur ce dont traite le présent ouvrage. Enrichi d'un index et d'une bibliographie, le plan s'ordonne autour de trois parties : "Droit administratif, État et management ", "Droit administratif, État et Territoire(s) ", "Droit administratif, État et dissension ". C'est dire qu'en l'ouvrant, on ne peut espérer $\mathrm{y}$ trouver certains thèmes classiques du droit

38. Xavier DupRE De Boulois et Martine KAluszYINSKI, Le droit en révolution (s). Regards sur la critique du droit des années 1970 à nos jours, vol. 23, coll. " Droit et société ", Paris, L.G.D.J., 2011. 
administratif. Et L'État $d u$ droit administratif constitue l'aboutissement d'un programme de recherche entamé avec l'écriture de La Constitution imaginaire de l'administration publiée en $2008^{39}$ suivi de la rédaction Des mises en scène de la décentralisation en 200940. Chacun de ces ouvrages rassemble autour de thèmes communs les principaux travaux de l'auteur. Il s'agit en réalité de bien plus qu'une simple compilation d'articles, puisque l'auteur n'hésite pas à en approfondir certains points ou à $\mathrm{y}$ ajouter des réflexions clairement novatrices. Le propos tourne néanmoins autour de thématiques chères à l'auteur : "territoires/décentralisation ", "service public/management " et "mutations de l'action publique". Avec L'État du droit administratif, Jacques Caillosse propose plusieurs figures de l'État dont chacune rend compte des transformations profondes auxquelles il se trouve confronté à différents niveaux (local ou central).

Si Jacques Caillosse n'est bien entendu pas le seul à le faire, il est incontestablement l'un des rares à persévérer dans le chemin étroit d'une littérature critique sur le droit administratif, au risque parfois de se tenir en marge d'une certaine doxa universitaire. Quelques considérations méthodologiques sont alors nécessaires pour mieux en saisir l'intérêt. Séparons-nous un moment de l'ouvrage pour mieux y revenir.

Qu'entendre par l'intitulé "(Re)penser le droit administratif "? Si l'on s'en tient à l'ouvrage éclairé par d'anciens travaux ${ }^{41}$ de l'auteur, (re)penser le droit administratif consiste d'abord à "faire entrer dans le champ assigné au droit

39. Jacques CAIllosse, La constitution imaginaire de l'administration: recherche sur la politique du droit administratif, coll. "Les voies du droit ", Paris, P.U.F., 2008.

40. Jacques CAILlosse, Les " mise en scène "juridiques de la décentralisation: sur la question du territoire en droit public français, vol. 52, coll. "Droit et société ", Paris, L.G.D.J., 2009.

41. Jacques CAILlosse, "Sur les enjeux idéologiques et politiques du droit administratif. Aperçu du problème à la lumière du "changement" ", (1982) 208 La Revue administrative 361, 361-368. 
administratif des questions délaissées, la plupart du temps par les juristes de doctrine " (p. 22). Essayons d'aller plus loin. Il y aurait à cet égard quelque vanité à opposer, dans le champ du droit administratif, "penseurs " et "techniciens" ou, pour employer l'expression restée célèbre de Rivero, "faiseurs de systèmes "42. Disons-le tout net : cette opposition-là n'est nullement pertinente pour l'analyse des discours doctrinaux en droit administratif. Penser le droit administratif passe nécessairement par une compréhension des normes et des énoncés propres à la discipline. Or, c'est en partant d'un tel prérequis que le titre du présent article " $(\mathrm{Re})$ penser le droit administratif " se révèlera - du moins l'espèrera-t-on - utile d'un point de vue doctrinal. Penser le droit administratif ne peut être dissocié de la manière d'aborder le discours objet - droit administratif - en partant du principe qu'il désigne l'ensemble des normes que l'on suppose propres au champ de cette discipline.

À partir de ce point de vue, il est possible en effet de tenter une présentation schématique des manières de faire la "doctrine " universitaire en droit administratif. La plus répandue privilégie deux mots : " exégèse " - au sens de commentaire de décisions et "systématisation " - au sens de tentative de mise en ordre d'une ou plusieurs grandes catégories du système juridique ${ }^{43}$. Les résultats de cette démarche sont à plus d'un titre fructueux, surtout lorsqu'il s'agit d'évoquer "l'européanisation "44 ou la

42. Jean RIVERO, Apologie pour les faiseurs de systèmes, Paris, Dalloz, 1951, chronique p. 99-102. Pour une tentative de présentation de ce "tournant " technicien de la doctrine: Anne-Laure GIRARD, La transformation historique de la théorie de l'acte administratif unilatéral, Paris, Dalloz, 2013, p. 8-14 (préface J-J. Bienvenu).

43. Telle est l'approche proposée par exemple par Marcel Waline : Marcel Waline, Traité élémentaire de droit administratif, 6e éd., Paris, Sirey, 1950.

44. Entre autres : Jean SIRINELLI, Les transformations du droit administratif par le droit de l'Union européenne. Une contribution au droit administratif européen, Paris, L.G.D.J., 2011 (préface Y. Gaudemet); Jean-Bernard AuBY (dir.), L'influence du droit européen sur les catégories du droit public, Paris, Dalloz, 2010. 
" constitutionnalisation "45 du droit administratif, par exemple. On ne peut dénier à cette manière de faire, communément qualifiée de dogmatique ${ }^{46}$, une quelconque pensée, même si la démarche comporte parfois des limites évidentes. Les spécialistes ${ }^{47}$ l'ont déjà fait savoir à maintes reprises : le champ du droit ne peut se concevoir sans la possibilité d'un travail de systématisation destiné au pouvoir, aux praticiens et à la doctrine universitaire elle-même lorsqu'elle s'adresse à un public d'étudiants.

Toute autre est la démarche qui cherche à comprendre les raisons d'une telle domination technicienne. Les réponses sont complexes et encore rares du côté des juristes ${ }^{48}$. Jacques Caillosse évoquait l'institution de l'agrégation - comprise comme un rituel anthropologique -, laquelle ne favoriserait nullement l'essor d'une pensée pluraliste ${ }^{49}$. Le sujet est vaste et, reconnaissons-le, n'a pas à ce jour fait l'objet d'une étude empirique déterminante, en dehors de quelques prises de position ${ }^{50}$. De manière

45. Louis FAVOREU, "L'apport du Conseil constitutionnel au droit public ", (1982) 13 Pouvoirs 17, 17-26; Louis FAVOREU, "La constitutionnalisation du droit ", Mélanges en l'honneur de R. Drago. L'unité du droit, Paris, Economica, 1997, p. 25-42.

46. Jacques Chevallier, Doctrine juridique et science juridique, vol. 50, coll. "Droit et société ", Paris, L.G.D.J. 2002, p.103-119.

47. Par exemple : Jacques CaILlosse et Olivier Renaudie (dir.), Le Conseil d'État et l'Université : pluriel et singularité, coll. "Thèmes et commentaires ", Paris, Dalloz, 2015; ASSOCIATION FRANÇAISE POUR LA RECHERCHE EN DROIT ADMINISTRATIF, La doctrine en droit administratif, coll. "Colloques \& débats ", Paris, Litec, 2010.

48. Olivier BEAUD, "L'œuvre de Gaston Jèze signifie-t-elle un repli de la doctrine publiciste française sur la technique juridique? ", (2013) 11 Juspoliticum, en ligne : <http://www.juspoliticum.com/L-oeuvre-deGaston-Jeze-signifie-t.html> (consulté le 19 décembre 2015).

49. Jacques CAILlosse, "Quel droit administratif enseigner aujourd'hui? ", (2002) 329 Revue administrative 454, 460-461. Une démonstration plus détaillée peut être consultée dans l'ouvrage de Christophe Charle: Christophe CHARLE, La république des universitaires, 1870-1940, coll. "L'univers historique ", Paris, Seuil, 1994, p. 246-254.

50. Thierry LE BARS, Agrégation de droit privé et de sciences criminelles : libres propos pour la suppression de "l'épreuve de 24 heures ", Paris, Dalloz, 2004, p. 4; Jean-Marie CARBASSE, "L'agrégation des facultés de droit ", 
complémentaire à l'intuition de Jacques Caillosse, on pourrait alors risquer l'hypothèse suivante : la technicité actuelle du droit administratif proviendrait moins de la domination d'un certain type de "positivisme "51 que de l'alliance objective entre une doctrine et les acteurs dominants du système juridictionnel

(2009) 2 R.D.P. 300, 300-319; Fabrice MEllerAY, "Les concours nationaux d'agrégation de l'enseignement supérieur ", (2013) 2 A.J.F.P. 70, 70-74; Olivier BEAUd et Rémy LIBCHABER, "Où va 1'Université? Les chemins de la liberté ", (2014) 49 La Semaine Juridique - Édition Générale 1264; Didier TRUCHET, "La réforme du statut des universitaires: l'agrégation et le reste. À propos du décret du 2 septembre 2014 ", (2014) 40 La Semaine Juridique - Édition Générale 983; Muriel FABRE-MAGNAN, "La réforme du statut des universitaires ", (2014) 38 Recueil Dalloz 2232; Olivier BEAUD, "Libres propos sur le concours d'agrégation du supérieur ", (2015) 16 A.J.D.A. 897, 920-927; on se reportera en outre à la dernière livraison de la revue Commentaire: Pierre Delvolve, Pierre Larouche, Calixto S. Filho et Edouard Lemoalle, "Sur la formation des juristes en France ", (2015) 152 Commentaire, 606-628.

La prudence s'impose à ce stade, même si l'héritage de Bourdieu, les mutations accélérées que connaît aujourd'hui l'université française ou la récente mise à mort de l'agrégation des sections 05 ou 06 au nom du pluralisme de la pensée en science économiques et de gestion rendent plus que jamais légitime une telle piste de réflexion : Pierre BouRdiEu et Jean-Claude PASSERON, Les héritiers: les étudiants et la culture, Paris, Éditions de Minuit, 1964; Pierre Bourdieu, Homo Academicus, coll. "Le sens commun ", Paris, Éditions de Minuit, 1984; Benjamin Elman, Civil Examinations and Meritocracy in Late Imperial China, Cambridge, Harvard University Press, 2013; L'avenir des sciences économiques à l'université en France, remis à Madame la Ministre de l'Enseignement supérieur par Monsieur Pierre Cyrille Hautcoeur, président de l'EHSS, Paris, 5 juin 2014, p. 6, 13, 30-31; Décret no 2014-997 du 2 septembre 2014 modifiant le décret $n^{\circ} 84-431$ du 6 juin 1984 fixant les dispositions statutaires communes applicables aux enseignants-chercheurs et portant statut particulier du corps des professeurs des universités et du corps des maîtres de conférences, J.O. 4 septembre 2014, art. 53; Erik NEvEU, Rapport du président du jury d'agrégation de science politique, 20142015, en ligne : <http://www.afsp.info/omasp/agregation/rapportagreg 20142015.pdf> (consulté le 14 octobre 2015).

51. Jacques CAILlosse, Introduire au droit, 2e éd., coll. "Clefs ", Paris, Montchrestien, 1995, p. 15. On pense ici au positivisme idéologique tel que défendu par exemple par Scarpelli; Uberto SCARPELLI, Qu'est-ce que le positivisme juridique?, coll. "La pensée juridique moderne ", Paris/Bruxelles, Bruylant, L.G.D.J., 1996, préface. 
français, le Conseil d'État ou la Cour de cassation. La composition du jury d'agrégation en serait peut-être le symptôme. La technicité d'un droit administratif pris dans un réseau de contraintes européen et international rejaillit nécessairement sur le discours doctrinal. Or, il semble que la légitimité d'une telle doctrine dépend de sa capacité à réduire les antinomies générées par le système juridique dans lequel elle s'insère. L'explication serait dès lors moins à rechercher dans une opposition entre juristes "techniciens " et "penseurs " que dans le mode de production des élites universitaires dans les facultés de droit52. Une question se pose à cet égard : comment s'est imposée dans les représentations des juristes universitaires la figure dominante du "jurisconsulte arrêtiste "53? Le développement d'une socio-histoire interrogeant les modes de production de la doctrine juridique permettra peutêtre de saisir les causes de cette domination technicienne qui caractérise aujourd'hui la production doctrinale en droit

52. On pense, en économie, aux travaux de David Colander: David Colander, The Making of an Economist, Redux, Princeton, Princeton University Press, 2008.

53. En partant de "types " à la manière de Weber, Guillaume Richard propose plusieurs modèles dominants de conceptualisation du droit public qui mériteraient à eux seuls un travail complémentaire du sien (rappelons que son sujet portait sur le droit public sous la troisième République et non sur la manière dont s'est imposé la figure du juriste technicien) permettant de retracer la manière dont s'est imposé ce que l'on désigne usuellement sous le terme de juriste technicien. Guillaume RICHARD, Enseigner le droit public à Paris sous la troisième République, coll. " NBT ", Paris, Dalloz, 2015, p. 345-389. Différemment mais dans le même sens : Marc Milet, Les professeurs de droit citoyens. Entre ordre juridique et espace public, contribution à l'étude des interactions entre les engagements et les débats des juristes français (1914-1945), thèse de doctorat, Paris, Science politique, Paris II, 2000. 

l'influence de la réforme dont est issu le décret du 27 mars 195455.

On recense ces dernières années une multiplication des travaux universitaires tentant d'opérer un retour aux fondements du droit public et privé56. À ces travaux, s'ajoutent plusieurs tentatives de penser l'évolution du droit administratif à travers les mutations sociales et juridiques qui sont les siennes57. Dans le

54. C. CHARLE, préc., note 49; Jean-Louis HALPERIN (dir.), Paris, capitale juridique (1804-1950). Études de socio-histoire sur la faculté de droit de Paris, Paris, Rue d'Ulm, 2011; Guillaume SACRISTE, La République des constitutionnalistes. Professeurs de droit et légitimation de l'État en France (1870-1914), Paris, Presses de Sciences Po, 2011; G. RICHARD, préc., note 53.

55. Dans ce sens: Frédéric AUDREN et Jean-Louis HALPÉRIN, La culture juridique française; entre mythes et réalités, $X I X^{e}-X X^{e}$ siècle, Paris, C.N.R.S., 2013, p. 224-234; Cédric Moreau DE Bellaing, Un bon juriste est un juriste qui ne s'arrête pas au droit. Controverses autour de la réforme de la licence de droit de mars 1954, vol. 83, coll. "Droit et société ", Paris, L.G.D.J., 2013, p. 83-97.

56. On prendra, pour illustrer le propos, les récentes rééditions de textes classiques, par exemple dans les collections "tiré à part ", "Rivages du droit " ou "Anthologie du droit " publiées chez les éditeurs Dalloz et LGDJ. Entre autres: Léon Aucoc et Benoît Plessix, La juridiction administrative et les préjugés, coll. "Tiré à part ", Paris, Dalloz, 2012. Ou encore : Baptiste Bonnet et Pascale Deumier, De l'intérêt de la summa divisio droit public- droit privé?, coll. "Thèmes et commentaires ", Paris, Dalloz, 2010.

57. Gérard Timsit, Archipel de la norme, coll. "Les voies du droit ", Paris, P.U.F., 1997; Jacques CHEVAlLIER, "Vers un droit post-moderne? Les transformations de la régulation juridique", (1998) 3 R.D.P. 659, 659714; André-Jean ARNAUD, Entre modernité et mondialisation. Cinq leçons d'histoire de philosophie du droit et de l'État, vol. 40, coll. "Droit et société ", Paris, L.G.D.J., 1998; Chales-Albert MORAND, Le droit néomoderne des politiques publiques, vol. 26, coll. "Droit et société ", Paris, L.G.D.J., 2000; André-Jean ARNAUD, Critique de la raison juridique : entre mondialisation et post-mondialisation, t.2, vol. 37, coll. "Droit et société ", Paris, L.G.D.J., 2003; André-Jean ARNAUD, Entre modernité et mondialisation. Leçons d'histoire de la philosophie du droit, vol. 20, coll. "Droit et société ", Paris, L.G.D.J., 2003; Jacques ChEVAllier, L'État post-moderne, 3e éd., vol. 35, coll. "Droit et société ", Paris, L.G.D.J., 2008. 
même temps, on observe une spécialisation croissante du droit public. Le droit administratif n'est pas en reste : la sophistication des raisonnements mis en œuvre par le juge ne cesse de gagner du terrain, au point qu'elle nécessite désormais une vigilance constante - pour ne pas dire totale - si l'on ne veut pas se sentir dépassé par "l'actualité ". Un tel écart n'est pas toujours facile à maintenir. De fait, les limites de l'approche technicienne apparaissent au grand jour. L'obsession d'actualiser sans cesse un droit positif dont les raisonnements sont de plus en plus complexes à comprendre au regard de la porosité grandissante des ordres juridiques ne permet plus toujours de prendre du recul sur la matière 58 . Pire encore : elle fait du juriste universitaire le débiteur perpétuel de l'agenda - et donc nécessairement des politiques jurisprudentielles - du Conseil d'État, de la Cour de justice de l'Union européenne ou de la Cour européenne des droits de l'homme. C'est précisément ce qu'évoquait déjà Jacques Caillosse dans son article consacré à l'enseignement du droit administratif59 à propos du fossé séparant le droit des professeurs et le droit des praticiens ${ }^{60}$. Au reste, c'est bien ce dont semble témoigner l'évolution contemporaine des principaux manuels : les traités, devenus rares ${ }^{61}$, laissent place aux "précis "62 ou à des

58. On fera remarquer que le phénomène n'est pas propre au droit administratif. Une partie de la doctrine en droit de l'Union européenne l'a aussi exprimé en particulier dans l'ouvrage suivant: Ulla NEERGAARD, Ruth Nielsen et Lynn Roseberry, European Legal Method. Paradoxes and Revitalization, Copenhagen, DJØF Publishing, 2011.

59. Jacques CAILlOSsE, préc., note 49, p. 343-358 et 454-472.

60. Id., p. 345. Dans le même sens : Jean-Jacques BIENVENU, "Remarques sur quelques tendances contemporaines de la doctrine en droit administratif ", (1985) 1 Droits 135, 153-160.

61. Pascale Gonod, Fabrice Melleray et Philippe YolKa, Traité de droit administratif, coll. "Traités Dalloz ", Paris, Dalloz, 2011, où les auteurs affirment dans l'avant-propos : "Sans avoir la prétention de livrer une reconstruction scientifique du droit administratif français, ni d'ordonner ce que d'aucuns tiennent pour un inextricable chaos, ils [les auteurs] souhaitent apporter leur pierre à la réflexion sur les recompositions en cours (...) Par son caractère pluriel et la trentaine d'auteurs réunis, le présent Traité rompt ainsi avec une solide tradition de la doctrine française de droit administratif, où les solistes - plus rarement les 
synthèses rigoureuses exposant les principes essentiels qui gouvernent la matière63. Dans ce contexte, on mesure à quel point penser le droit administratif au sens où nous y invite Jacques Caillosse s'avère à plus d'un titre salutaire d'un point de vue intellectuel. Car la prise de distance avec le droit administratif passe nécessairement par une mise en doute de la façon de faire la doctrine selon la manière de la doxa majoritaire. Un tel point de vue n'est pas toujours facile à maintenir : en effet, il implique parfois de n'être considéré ni tout à fait comme un "vrai " juriste, ni tout à fait comme un pur sociologue.

Ajoutons que penser le droit administratif au sens où Jacques Caillosse nous y invite ne signifie nullement prétendre reconstruire le droit administratif sur de nouvelles bases conceptuelles, à la manière de ce qui a été entrepris au début du $\mathrm{XX}^{\mathrm{e}}$ siècle ${ }^{64}$. Il ne s'agit pas non plus, derrière la rhétorique d'une supposée crise du droit administratif, de se complaire dans une forme de nostalgie à l'égard de la " belle époque " et des "écoles "65. Le défi de penser le droit administratif contemporain consiste, si l'on suit Jacques Caillosse, à saisir la complexité contemporaine de la matière dans sa totalité. Cette complexité qui lui est inhérente traduit quelque chose de plus profond sur les transformations de l'État. Derrière ce mot se cachent plusieurs

formations de chambre - dominent, aux dépens de la musique d'orchestre " (p. 6-7).

62. Pierre-Laurent FRIER et Jacques PETIT, Précis de droit administratif, 9e éd., coll. "Domat ", Paris, L.G.D.J., 2014.

63. Par exemple : Bertrand SEILlER, Droit administratif, 5e éd., coll. "Champ. Université ", Paris, Flammarion, 2014 (2 tomes); Didier TRUCHET, Droit administratif, 5e éd., coll. "Thémis ", Paris, P.U.F., 2013. Sur cette question: Anne-Sophie CHAmвоsт (dir.), Histoire des manuels de droit. Une histoire de la littérature juridique comme forme du discours universel, coll. "Contexte, culture du droit ", Paris, L.G.D.J., 2014, p. 9-24.

64. Par exemple : Maurice HAURIOU, Précis de droit administratif et de droit public, 11e éd., Paris, Sirey, 1927; Maurice Hauriou, Cours de science sociale: La science sociale traditionnelle, Paris, Larose, 1896; Gaston JEze, Principes généraux du droit administratif, 3e éd., vol. 6, Paris, GiardL.G.D.J., 1925.

65. Jacques CHEvallier, "La fin des écoles? ", (1997) 3 R.D.P. 679, 679-700. 
réalités fort différentes: celles relatives aux interventions administratives ou encore celles des légitimités inhérentes à son action. La tâche de penser le droit administratif suppose de construire un objet sans le réduire arbitrairement au contentieux. Car penser le droit administratif avec Jacques Caillosse, c'est se risquer à penser tout le droit administratif : celui du juge, mais aussi celui de l'administration. Un tel objet ne peut être appréhendé qu'en mobilisant une ouverture pluridisciplinaire en direction des politiques publiques et de la sociologie politique. Penser le droit administratif, c'est finalement reconstruire l'unité d'une authentique science de gouvernement66 dans un contexte épistémologique de fragmentation disciplinaire entre des sciences sociales - sciences politiques et sociologie - qui s'intéressent aux politiques de l'administration et les juristes - gagnés eux aussi par l'hyperspécialisation - qui se concentrent principalement sur le droit du juge. Tel est selon nous l'apport majeur de l'ouvrage que les critiques que nous formulerons ne remettent nullement en cause.

\section{II- Une réflexion pluridisciplinaire de juriste}

De quel(s) "État(s) " Jacques Caillosse nous entretient-il? Car l'ouvrage n'est pas, à proprement parler, celui d'une théorie de l'État au sens où l'entendent communément les spécialistes de la discipline, c'est-à-dire un discours sur "l'État-Nation "67. Il est plutôt question ici d'interroger ce que " (...) nous enseigne le droit administratif sur l'État "68 et, plus précisément, sur les figures de l'État que, pour le dire avec les mots de Jacques Caillosse, le droit

66. Sur la cartographie d'un tel programme de recherche : Renaud PAYRE et Rachel VANNEuville, "Les habits savants du politique". Des mises en forme savantes $\mathrm{du}$ politique à la formation des sciences de gouvernement ", (2003) 53 R.F.S.P. 219, 219-235.

67. Cette expression de Bénoît est reprise par Olivier Beaud dans sa contribution au Traité de droit administratif. Elle réfère aux normes qui s'appliquent à la constitution, à la loi ou au décret: Olivier BEAUD, "L'État ", dans Pascale GonoD, Fabrice Melleray et Philippe YolKa (dir.), Traité de droit administratif, Paris, Dalloz, 2011, à la page 229.

68. Selon là encore la formulation qu'en propose Olivier Beaud : Id., p. 208. 
administratif "met en scène" (p. 26-28). En somme, l'auteur convoque le discours du juge administratif pour en extraire les transformations contemporaines des politiques publiques. Derrière la technicité vertigineuse qui le caractérise et dont les juristes demeurent friands au point parfois de l'ériger en "veau d'or ", le droit administratif nous parle aussi des transformations de fond que traverse l'action administrative contemporaine. Voilà l'angle mort, souvent délaissé par la doctrine contemporaine, sur lequel l'auteur nous invite à réfléchir sans pour autant prétendre à l'exhaustivité intellectuelle. La démarche méthodologique est clairement d'inspiration sociologique, analysant les normes et les discours sur les normes comme un discours-objet69. À son habitude, l'auteur convoque les réflexions plurielles de Pierre Legendre, Pierre Bourdieu, Bruno Latour, ou plus occasionnellement de Charles Eisenmann, afin de proposer une lecture originale du droit administratif.

Il convient dès à présent de mieux préciser l'affirmation selon laquelle la démarche de Jacques Caillosse serait d'inspiration sociologique. Par là, il ne s'agit certainement pas de disqualifier la qualité de juriste de l'auteur. Bien souvent, hélas, c'est en prenant prétexte de l'originalité d'une telle méthode que l'on en vient à en marginaliser le point de vue par la phrase désormais magique : "c'est un travail très intéressant, mais ce n'est pas du droit ". Sous l'emploi d'un tel obiter dictum, que l'on voudrait dès à présent prévenir, se cache la tentation d'éviter d'interroger les présupposés de l'emprise du droit administratif tel qu'il se pratique majoritairement au sein de la doctrine universitaire. Ainsi, L'État $d u$ droit administratif est un livre qui s'adresse en premier lieu aux spécialistes du droit public.

Outre qu'il interroge la pratique usuelle de la doctrine, l'ouvrage vient enrichir la connaissance scientifique du fonctionnement des institutions administratives. Plus

69. Véronique Champeil-Desplats, Méthodologies du droit et des sciences du droit, coll. "Méthodes du droit ", Paris, Dalloz, 2014, p. 24-28. 
fondamentalement, L'État $d u$ droit administratif apporte une réflexion sur ce que nous dit le droit administratif sur les transformations de l'État. Ces transformations ne peuvent être appréhendées qu'en prenant au sérieux l'infra-droit produit par l'administration - circulaires, directives, instructions, etc. - et les pratiques qui l'accompagnent. En ce sens, le propos n'établit aucune frontière entre les politiques publiques, la sociologie politique et les institutions administratives telles qu'elles sont traditionnellement enseignées dans les facultés de droit. De la même manière, les aspects théoriques sont étroitement mêlés aux préoccupations techniques, ce qui rend stimulante la lecture entière de l'ouvrage. Les développements qui vont suivre s'inspirent de cette trame féconde. L'articulation de propos théoriques nourris d'analyses techniques ne doit, dès lors, pas surprendre. Cela précisé, entrons dans le sujet.

La démonstration qui sous-tend L'État $d u$ droit administratif est essentiellement juridique. Car Jacques Caillosse est avant tout un juriste qui se livre à une analyse très fine des phénomènes juridiques. L'analyse du territoire qu'il propose l'illustre. On pourrait en résumer ainsi la trame: la décentralisation s'opère et ne se pense qu'à travers les cadres et les formes de l'État central. On a encore en tête les développements passionnants qu'il consacre au déféré préfectoral (p. 157-179). Le lecteur y apprend les étapes qui ont conduit au contrôle de légalité animé par la volonté d'éviter

le fameux face à face du préfet et de ses notables, parce qu'il avait fini par y voir, lui aussi, l'expression condensée d'un centralisme aussi peu compatible avec les exigences de la démocratie locale qu'avec celles de l'efficacité de l'action publique (p. 160).

C'est précisément la réinstallation de cette ancienne logique qui a été validée a posteriori par le Conseil d'État dans son rapport public de 1993 : Décentralisation et ordre juridique. Ce que donne à voir ce rapport, c'est que le juge administratif n'entrevoit pas la décentralisation de la même manière que le législateur de 1982. Le rapport public de 1993 remet l'État au cœur de la politique de 
décentralisation là où il s'agissait précisément de neutraliser l'influence des rapports entre le préfet et ses notables. Juridique, la démonstration de Jacques Caillosse sur le déféré l'est assurément. Son objet n'est nullement différent de celui dont se sert communément la doctrine administrativiste, à ceci près que Jacques Caillosse en propose une interprétation originale. Là réside la force d'un propos qui ne se limite pas à chercher une cohérence entre plusieurs normes ou énoncés parfois contradictoires. En ce sens, la méthode de l'auteur est davantage mue par le désir de " comprendre " que "d'ordonner ", car Jacques Caillosse s'attache à mettre en évidence les réalités sociales et politiques que le droit administratif est supposé saisir.

L'intention du législateur a bien été à l'origine d'instaurer une décentralisation totale dans laquelle disparaitrait la tutelle des Commissaires de la République, s'agissant du contrôle a priori des actes pris par les collectivités territoriales. Que s'est-il passé pour que cette tutelle se maintienne malgré tout? Le récit juridique de cette histoire comporte trois épisodes. Le premier se caractérise par l'intervention du Conseil constitutionnel qui, dans sa décision du 25 février $1982^{70}$, rappelle à l'ordre le législateur : "Considérant qu'il résulte des dispositions précitées de l'article 72 de la Constitution que si la loi peut fixer les conditions de la libre administration des collectivités territoriales, c'est sous la réserve qu'elle respecte les prérogatives de l'État (...) " ${ }^{71}$. Ce dernier est sommé de "revoir sa copie" (p. 161). Le second épisode caractérise la manière dont le Conseil d'État va réorienter la pratique du déféré préfectoral ${ }^{72}$ en s'émancipant de l'intention

70. Cons. const. 25 février 1982, Loi relative aux droits et libertés des communes des départements et des régions, DC. 1982.137.

71. Id., pt. 4.

72. Le déféré préfectoral s'entend du pouvoir que détient le préfet de par la loi de saisir le juge administratif d'une éventuelle illégalité d'un acte pris par une collectivité territoriale (commune, département, région). En effet, les actes des collectivités territoriales sont nécessairement transmis avant leur promulgation officielle au préfet. Comme l'a jugé la jurisprudence, cette saisine du juge par le préfet n'est pas automatique, ce qui donne parfois lieu à un jeu d'influence des préfets sur les 
initiale du législateur. Le résultat est désormais connu : les déférés préfectoraux sont peu nombreux. Quant au contrôle $a$ priori des actes, c'est-à-dire aux observations adressées à leurs auteurs, il reste dérisoire au regard des demandes transmises ${ }^{73}$. Mieux, en assimilant la procédure du déféré préfectoral à un simple recours pour excès de pouvoir, la jurisprudence administrative prend le parti, nous dit Jacques Caillosse, d'en banaliser l'originalité initiale. Un tel choix n'a pas été sans conséquence, ainsi que le démontre avec conviction l'auteur. La banalisation du déféré préfectoral a grandement contribué à maintenir l'ancien système clientéliste centre/périphérie. Le point d'orgue de cette banalisation s'achève avec les jurisprudences Brasseur et Préfet des Bouches-du-Rhône c. Commune de Belcodène $^{74}$ (p. 178) par lesquelles le Conseil d'État énonce que, dans le cadre du contrôle de légalité, le préfet n'est pas tenu de saisir le juge lorsqu'il est confronté à l'illégalité d'un acte.

La compréhension théorique qu'offre L'État du droit administratif sur la décentralisation rappelle à nouveau que "les juristes" - entendons, les discours de la doctrine universitaire sur la décentralisation - ne parviennent pas toujours à l'aborder sans convoquer la figure de l'État central. La décentralisation des juristes se conçoit dès lors comme un " espace de jeu dont dispose l'État vis-à-vis des collectivités territoriales (...) lui-même borné par les interprétations que le juge a données à la notion de libre administration" (p. 146). La pertinence de la représentation usuelle d'un tel discours sur la décentralisation doit par conséquent être interrogée. Certes, il faut reconnaître que les manuels autorisés n'ont jamais pris pour argent comptant

collectivités territoriales. On renvoie au rapport "Mercier " mentionné cidessous.

73. SENAT, Rapport d'information $n^{\circ} 447$ du 28 juin 2000 du Sénat réalisé au nom de la mission d'information par Monsieur Michel Mercier, p. 87-94, en ligne: <http://www.senat.fr/rap/r99-447-1/r99-447-11.pdf> (consulté le 18 décembre 2015).

74. Cons. d'Ét. 16 juin 1989, Préfet des Bouches du Rhône c. Commune de Belcodène, Rec. Cons. d’Ét., p. 512; Cons. d’Ét. 6 décembre 1999, Société Aubettes SA, n¹96403, R.F.D.A. 2000. 1242. 
l'analyse du phénomène ${ }^{75}$. Il n'en reste pas moins que s'en tenir à une analyse de la décentralisation purement normative et classiquement institutionnelle comporte des limites évidentes. La plus importante tient à l'étroitesse d'une vision binaire, selon les cas, du type : l'évolution de la décentralisation masque un retour lancinant de l'État ou, au contraire, l'évolution de la décentralisation tend vers une émancipation progressive des territoires sur le modèle fédéral. Entre ces deux extrêmes, point de salut; comme s'il n'était pas possible de penser au sein de l'État unitaire différents modèles d'organisation des territoires. Là encore l'étroitesse de la question présuppose une impossibilité de penser en dehors d'un modèle "jacobin" d'État unitaire orchestrant sa propre décentralisation.

Le récent débat sur les nouvelles régions n'a pas manqué d'illustrer ce dernier point. La fusion a d'emblée été dénoncée comme annonçant un risque de dérive fédéraliste dont l'Union européenne aurait tout intérêt à ce qu'elle se réalise ${ }^{76}$. La réalité est plus contrastée, puisque, à s'en tenir au projet actuellement discuté devant la représentation nationale, la création des grandes régions ne s'accompagne nullement d'un pouvoir accru de ces dernières dans le domaine financier. Comme le déplorait récemment un auteur : "Les élus ont dépensé beaucoup d'énergie à s'entredéchirer sur la carte, alors que l'essentiel, c'est le développement des territoires et la capacité politique à agir "77. Le

75. Par exemple: Henri OBERDOFF et Nicolas KADA, Les institutions administratives, 7e éd., coll. "Université ", Paris, Sirey, 2013, p. 168-170.

76. Voir entre autres: Patrick LE GALES et Christian LEQUESNE (dir.), Les paradoxes des régions en Europe, coll. "Recherches", Paris, La découverte, 1997; Christian BATAIILON, L'émergence du fait régional au sein de l'Union européenne: la coopération trans-frontalière comme stratégie de développement, coll. "Études", Perpignan, Presses Universitaires de Perpignan, 2002; Marie-Thérèse BITSCH (dir.), Le fait régional et la construction européenne, coll. "Organisation internationale et Relation internationale ", Bruxelles, Bruylant, 2003; Paul DiRKX, La concurrence ethnique: la Belgique, l'Europe et le néolibéralisme, coll. "Savoir/Agir ", Bellecombes-en-Bauges, Le croquant, 2012.

77. Romain PASQUIER, "Pour réussir la régionalisation, il faut créer un gouvernement régional ", interview, Acteurs publics, 24 mars 2015, en 
discours sur la régionalisation semble en effet en rester au stade des mots: en maintenant d'un côté les départements et les services déconcentrés au niveau régional tout en accentuant d'un autre côté le pouvoir des métropoles ${ }^{78}$, l'actuelle réforme territoriale confirme le caractère indépassable de la figure de l'État unitaire à la française.

L'usage fréquent par la doctrine du néologisme "déconcentralisation "79, en ce qu'il oppose déconcentration et décentralisation, ne rend pas toujours justice de ce qui se joue en matière de réforme territoriale. Du côté de l'État, la critique de la décentralisation constitue un argument commode pour contraindre les collectivités territoriales à la réforme souhaitée par le centre. La récente baisse des dotations financières en est un exemple topique. En s'arrogeant le monopole de la "raison budgétaire ", l’État, comme le montre Jacques Caillosse (p. 150151), se donne les moyens de proposer les formes de regroupements juridiques les plus admissibles - généralisation du modèle de communauté de communes, par exemple. Du côté des collectivités territoriales, le discours de la victimisation n'est, ainsi que le montre l'auteur, guère plus crédible. Les "élus locaux occultent parfois (...) certaines des caractéristiques essentielles du pouvoir décentralisé, et tout spécialement le fait que ce dernier est lui-même largement conçu sur le modèle étatique " (p. 152).

Cette même hypothèse anime les développements consacrés au thème de la différenciation territoriale (chapitre 7). L'invocation

ligne : <http://www.acteurspublics.com/2015/03/24/1-essentiel-est-decasser-la-verticalite-de-1-action-publique> (consulté le 20 avril 2015).

78. Loi $n^{\circ}$ 2014-58 du 27 janvier 2014 de modernisation de l'action publique territoriale et d'affirmation des métropoles: Michel DEGOFFe, "L'organisation des métropoles ", (2014) 3 R.F.D.A. 481.

79. Eisenmann parle aussi de "semi-décentralisation ": Charles EISENMANN, Centralisation, décentralisation, esquisse d'une théorie générale, Paris, L.G.D.J., 1948; Charles EISENMANN, "Les structures de l'administration ", dans Jean-Marie AuBY (dir.), Traité de science administrative, La Haye, Mouton \& Co., 1966, aux pages 261-318; Charles EISEnMANn, Cours de droit administratif, t.1, Paris, L.G.D.J., 1982, p. 157-300. 
de ce thème, aujourd'hui repris en cœur par certains acteurs politiques en matière de décentralisation, n'a rien de nouveau. La possibilité juridique pour l'État de déroger à l'égalité supposée entre territoires existait dès le début des lois de décentralisation : le Conseil constitutionnel en a légitimé sous certaines conditions la pratique. Or, ainsi que le montre Jacques Caillosse, le thème de la différenciation territoriale est le fruit d'une contradiction insoluble: celle de prétendre pouvoir adapter une norme aux spécificités d'un territoire alors que cette norme n'est pensée qu'au travers du droit de l'État central. Toute tentative d'envisager les choses différemment se heurte à la fausse alternative: État unitaire versus État fédéral.

D'un côté on cherche à desserrer un maillage territorial invariablement reproduit par la norme nationale, en valorisant les singularités du local; de l'autre on reconstitue ce maillage national par une décentralisation normative qui dissout le local (p. 193).

Ici, l'État "territorial " cède la place à l'État " manager " : celui qui gouverne à distance ${ }^{80}$ par l'élaboration d'une "standardisation des politiques publiques" (p. 192) à travers la LOLF, la RGPP puis la MAP. Si la loi permet l'expression de la différenciation, elle ne le permet que de manière relative et voulue par l'État central, qui n'hésite pas, si nécessaire, à s'emparer du discours sur l'inégalité des territoires afin de mieux légitimer une forme parfois masquée de recentralisation ${ }^{81}$.

La récente proposition législative relative à la possible fusion de Maisons de services au public et de la Poste en s'appuyant sur le réseau territorial de cette dernière est

80. Selon le titre qu'emprunte Jacques Caillosse à l'article de Renaud EPSTEIn, "Gouverner à distance. Quand l'État se retire des territoires ", (2005) Esprit 96, 96-111.

81. Matthieu CONAN, "L'autonomie financière des collectivités territoriales ", dans Trente ans de décentralisation, Paris, A.J.D.A., 2012, p. 759-763. 
exemplaire de la thèse évoquée ${ }^{82}$. Ce sont bien les contraintes propres à l'État actionnaire, dans un contexte d'ouverture à la concurrence, qui sont à la base de la transformation des bureaux locaux de poste en Maisons de services au public. Autrement dit, c'est l'État qui pense la différenciation territoriale, sans nécessairement associer les territoires à la réflexion. Telle est la grille de lecture critique qu'il convient d'appliquer lorsque, dans un discours prononcé à Laon le 13 mars 2015, le premier ministre annonçait la création d'un comité interministériel consacré aux ruralités. Parmi les mesures, le gouvernement annonçait la création de 1000 Maisons de services au public à l'horizon $2016^{83}$. On assiste, ce faisant, à une nouvelle manière de penser l'action dans laquelle l'État se retire des territoires "pour mieux les gouverner à distance "84. Aussi, convient-il, pour comprendre la manière dont évolue l'administration des territoires, de se tourner vers le concept foucaldien de "gouvernementalité ", dont la littérature sur les politiques publiques use à volonté (chapitre 8). Il permettrait, selon l'auteur, de mieux saisir la complexité des mutations administratives qui caractérisent aujourd'hui l'administration territoriale.

Il ne s'agit là que d'un simple aperçu sur un ouvrage intellectuellement très dense. Certaines parties sont le fruit de réflexions abouties : le chapitre consacré à la "surdétermination économique du service public" ou la partie intitulée "Droit administratif, État et territoires(s) ", entre autres. D'autres

82. Assemblee nationale, Rapport présentée le 14 janvier 2015 par Jean Launay pour la Commission des finances, de l'économie générale et du contrôle budgétaire sur les conditions de maintien du service public dans les territoires et la contribution que la Poste pourrait y apporter, en ligne : <http://www.assemblee-nationale.fr/14/rap-info/i2495.asp> (consulté le 22 mars 2015).

83. Manuel VALLS, "Les ruralités, avec toute la diversité des paysages, des métiers, des traditions, sont autant de chances pour la France ", (2015) Actualité, en ligne: <http://www.gouvernement.fr/les-ruralites-avectoute-la-diversite-des-paysages-des-metiers-des-traditions-sont-autantde-chances $>$ (consulté le 22 mars 2015).

84. R. EPSTEIN, préc., note 80, p. 111. 
développements proposent, en revanche, des pistes de recherche. Ainsi, lorsque Jacques Caillosse évoque la création d'un champ d'études consacré au droit de la performance, qu'il interprète avec la surdétermination économique du service public comme une manifestation du déplacement de la "rationalité juridique ", de quelles manières approfondir cette hypothèse d'un déplacement de la rationalité juridique? Quel contenu donner à cette dernière expression? Signifie-t-elle qu'il existerait une rationalité spécifique au droit? Encore faudrait-il pouvoir le démontrer, sachant que l'auteur n'en précise pas davantage le contenu. Quant aux pistes de recherche évoquées relatives au "droit de la performance ", vers où s'orienter? Le management auquel est soumis l'ordre juridictionnel administratif lui-même, voire celui de la Cour des comptes, pourrait-il constituer un des objets de ce "droit du management " 85 ?

Comment les élites administratives se sont progressivement converties à la "culture " de l'évaluation et du management ${ }^{86}$ ? L'étude de l'ordre administratif, c'est-à-dire des modes de travail et d'évaluation quotidiens des juges du fond ainsi que les relations de ces derniers avec le corps des membres du Conseil d'État, pourrait-elle constituer à cet égard une piste de réflexion fructueuse? Il est permis de penser que l'étude de l'ordre juridictionnel administratif et judiciaire constituerait peut-être un terrain privilégié de ce "droit de la performance " dont parle Jacques Caillosse au chapitre 2. Une autre thématique non moins originale est l'analyse des modes non juridictionnels de résolution des conflits internes à l'administration, encore peu répandue dans la doctrine de droit administratif (p. 255-266).

85. Elisa CHELle, "Une politique de récompense dans la magistrature. Le cas des primes de rendement des magistrats de cours d'appel et de cassation ", (2011) 79 Droit et Société 407, 407-427.

86. Une piste de réflexion peut être consultée dans la livraison de 2012 de la revue Actes de la recherche en sciences sociales: Julie GERVAIS, "Les sommets très privés de l'État", (2012) 194 Actes de la recherche en sciences sociales 4, 4-21. 


\section{III- Un regret et deux critiques}

Que l'on nous permette d'exprimer à ce stade un regret et deux critiques. Le regret tout d'abord : L'État du droit administratif reste étrangement silencieux sur l'Europe en général et sur le droit européen en particulier - qu'il s'agisse du droit de la Convention ou du droit de l'Union européenne. L'auteur prend soin d'évacuer ce point dès l'introduction (p. 33). Certes, l'évocation du droit européen apparaît en creux dans l'ensemble du propos - la surdétermination économique du service public, par exemple -; on ne peut pour autant se satisfaire de ce silence à l'heure de l'émergence d'une littérature qui se consacre au droit administratif européen $^{87}$. Il aurait été intéressant d'entendre l'auteur sur la manière dont, en matière de pratiques administratives, une transformation de l'action publique s'opère sous l'influence de l'Europe. Si l'article $6 \$ 1$ reste abondamment commenté par les juristes, rien n'est dit sur la redéfinition des politiques publiques qu'engendrent les obligations liées au procès équitable dans les administrations concernées.

Un exemple récent: lorsque le Conseil constitutionnel88 juge non conforme aux principes d'impartialité et d'indépendance

87. Benedict KingSBURY, Nico Krisch et Richard STEWART, "The Emergence of Global Administrative Law ", (2005) 3-4 Law \& Contemporary Problems 1, 1-356; Matthias RUfFERT (dir.), The Transformation of Administrative Law in Europe, Munich, European Law Publisher, 2007; Jurgen ScHWARzE, Droit administratif européen, coll. "Droit administratif ", Bruxelles, Bruylant, 2009; Jurgen SchwARZE, L'état actuel et les perspectives du droit administratif européen, coll. "Droit administratif ", Bruxelles, Bruylant, 2010; Clémentine BoRIEs (dir.), Un droit administratif global?, coll. "Cahiers internationaux ", Paris, Pedone, 2012; Paul CRAIG, European Admnistrative Law, Oxford, Oxford University Press, 2012; Daniel MockLE, "Le débat sur les fondements du droit administratif global ", (2012) 53 C. de D. 3, 3-48; Jean-Bernard AuBY et Jacqueline DutHeIl DE LA ROCHERE, Traité de droit administratif européen, coll. "Droit administratif ", Bruxelles, Bruylant, 2014; Emilie CHEvalier, Bonne administration et Union européenne, coll. "Droit administratif ", Bruxelles, Bruylant, 2014 (préface O. Dubos).

88. Cons. const. 8 juin 2012, Christian G, Rec. Cons. const., p. 50. 
la composition des Commissions départementales d'aide sociale, quelle conséquence en tirer du point de vue de l'organisation administrative? Plutôt que d'envisager une réforme d'ensemble des juridictions sociales, l'Assemblée nationale, reprenant sur ce point le projet de loi présenté par le gouvernement, a décidé d'en complexifier le contentieux - par l'introduction d'un amendement relatif au recours administratif préalable obligatoire dont les juridictions administratives auraient eu à connaître. Fort heureusement, la rédaction de cet article a été retirée par un amendement sénatorial. Le problème n'en demeure pas moins latent: quelles politiques publiques l'État met-il en œuvre pour faire face aux exigences liées aux principes d'impartialité, qu'il s'agisse de juridictions ou d'autorités administratives indépendantes? Pour ce qui concerne les juridictions sociales, il semble que la réponse apportée s’inscrive dans le mouvement plus vaste d'un dispositif de "gouvernance des pauvres" 89 .

L'ouvrage évacue un peu vite, à notre grand regret, la littérature se réclamant du droit administratif global ${ }^{90}$ (Global Administrative Law). Fondé sur un ensemble de principes ("transparence ", "prévention des conflits d'intérêts", "responsabilité des administrations", soft law ${ }^{91}$ ), ce discours participe puissamment à justifier l'uniformisation de nombreuses

89. Titre de l'ouvrage d'Elisa Chelle : Elisa CHELle, Gouverner les pauvres. Politiques sociales et administration $d u$ mérite, Rennes, Presses Universitaires de Rennes, 2012.

90. Voir en ce sens : Mikhail XIFARAS, "Après les théories générales de l’État : le droit global?", (2012) 8 Juspoliticum, en ligne : <http://www.juspoliticum.com/IMG/pdf/JP8-Xifaras.pdf> (consulté le 26 avril 2015); Jean-Yves CHEROT et Benoît FRYDMAN (dir.), La science du droit dans la globalisation, coll. "Penser le droit ", Bruxelles, Bruylant, 2012.

91. On se demande dans quelle mesure le Conseil d'État s'inscrirait dans ce mouvement global: CONSEIL D'ÉTAT, Le droit souple, coll. "Études et documents", CONSEIL D'ÉTAT, Paris, La documentation française, 2013. Dans le même sens: Benjamin LAVERGNE, Recherche sur la soft law en droit public français, coll. "Les thèses de l'IFR ", Paris, L.G.D.J., 2013; ASSOCIATION HENRI CAPITANT, Le droit souple, coll. "Thèmes et commentaires ", Paris, Dalloz, 2008. 
pratiques administratives au sein d'instances comme l'OCDE, le FMI et des institutions européennes. Est-il possible d'établir un lien entre les réformes liées au nouveau management public et l'avènement d'un droit administratif global92? On voit émerger, depuis plus d'une vingtaine d'années, dans les politiques menées par le Conseil de l'Europe et l'Union européenne, une sémantique tirée des outils conceptuels dont se revendique le droit administratif global : "bonne gouvernance ", " conflits d'intérêts ", " lignes directrices ", " bonnes pratiques "93, etc. De quelle manière les droits de l'Union européenne et du Conseil de l'Europe convergent-ils en matière d'utilisation d'instruments communs de ces outils de gouvernance? À l'évidence, une culture et des intérêts communs émergent depuis plusieurs années entre les élites administratives et les institutions européennes ${ }^{94}$; s'y ajoute, même si l'on en parle moins, la création de réseaux transnationaux entre juges dont l'objectif vise à harmoniser un certain nombre de standards entre cours suprêmes ${ }^{95}$. La présentation usuelle de tout ceci est résumée par l'expression

92. Jacques ZILLER, "Le droit public, un outil de gestion publique moderne et non un frein à la réforme ", (2005) 71 Revue Internationale des Sciences Administratives 281, 281-292.

93. Par exemple : Convention pénale sur la corruption adoptée dans le cadre du Conseil de l'Europe du 27 janvier 1999; Convention civile sur la corruption adoptée dans le cadre du Conseil de l'Europe du 4 novembre; CONSEIL DE L'EUROPE, Résolution 90 (6) relative à un Accord partiel portant création de la Commission européenne pour la démocratie par le droit, en ligne : < < <ttp://www.venice.coe.int/WebForms/pages / $\mathrm{p}=01_{-}$ Presentation> (consulté le 20 mars 2015).

94. Cette culture commune a des racines anciennes: Antonin CoHEN, "De congrès en assemblées ", (2006) 18 Politique européenne 105, 105-125; Antonin COHEN, "L'autonomisation du "Parlement européen". Interdépendance et différenciation des assemblées parlementaires (années 1950 - années 1970) ", (2012) 85-6 Cultures et conflits 13, 13-33.

95. On pense encore ici à la Commission dite de Venise encore appelée "Commission européenne de la démocratie par le droit " créée par le Conseil de l'Europe en 1990 (Résolution 90 (6), préc., note 93) ou encore, s'agissant du droit de l'Union européenne, au programme de formation : EUROPEAN JUSTICE, La politique de formation judiciaire européenne, en ligne : <https://e-justice.europa.eu/content_the_european_judicial_ training_policy-121-fr.do $>$. 
large "d'européanisation " ou, pour ce qui concerne les juges, de "dialogue ", sans que l'on sache vraiment ce que recouvre clairement l'emploi de ces deux expressions. Or, il convient précisément de sortir du piège de "l'européanisation ", si par là on entend les relations entre les rapports de systèmes juridiques, afin d'en cerner plus spécifiquement les effets en terme de pratiques administratives.

D'un point de vue institutionnel, l'européanisation s'entend principalement de l'attractivité des politiques européennes dont la multiplication des agences, experts et autorités administratives indépendantes serait le symptôme. À cela, on ajoute, s'agissant plus spécifiquement du droit de l'Union européenne, que les rapports entre administrations nationales et européennes s'organiseraient de plus en plus sur le modèle d'une administration fédérale. Comment appréhender une telle influence sans, là encore, tomber dans le piège d'un simple transfert de culture administrative au profit de l'Union européenne? Nul ne doute que le modèle administratif européen exerce indéniablement une influence plus ou moins grande sur les administrations nationales; la question est ailleurs. Elle est dans le degré d'influence et d'attractivité de ce modèle européen. Topique de cette affirmation, l'application du droit de concurrence s'organise désormais en "réseau " en apparence coordonné par la Commission européenne. L'institutionnalisation de ce réseau nécessite cependant la mise en œuvre d'instruments divers et variés : vulgarisation d'un registre technocratique commun par le recours à une rhétorique économique, programme de formation des juridictions ou administrations nationales, etc. L'objectif affiché ici est la création d'une communauté épistémique commune, autrement dit, un ensemble d'acteurs qui partagent un ensemble de valeurs communes ${ }^{96}$. Pourtant, contrairement aux idées reçues, les rapports entre administrations nationales et européennes ne se posent pas en terme de gouvernance

96. Michaela Drahos, Frans VAN WAARDEN, "Courts and (Epistemic) Communities in the Convergence of Competition Policies ", (2002) 9 Journal of European Public Policy 913, 913-934. 
hiérarchique, mais bien de transferts partiels de culture administrative ${ }^{97}$. Bien plus, la création de ces réseaux, dont le droit de la concurrence n'a bien sûr pas le monopole ${ }^{98}$, engendre parfois des situations fort complexes où les autorités nationales intégrées dans le réseau poursuivent des intérêts divergents des administrations centrales ou des autorités décentralisées ${ }^{99}$.

C'est ce que donnent à voir les récents contentieux afférents aux organismes génétiquement modifiés ou celui des antennesrelais, lorsque le Conseil d'État fait primer la police spéciale sur la police générale100. Au-delà des débats relatifs à l'articulation des polices ou à la répartition contentieuse, il y a aussi la prise en compte d'un phénomène de "réseau " d'autorités nationales qui s'insère pleinement dans le fonctionnement d'une politique désormais menée au niveau européen; cette dernière ne peut souffrir d'éventuelles politiques nationales contraires. Autrement

97. Andy Smith, "Transferts institutionnels et politique de concurrence. Les cas communautaire, français et britannique ", (2013) 3 Gouvernement et action publique 415, 415-440.

98. On pense ici au secteur de l'énergie dont les régulateurs se structurent autour de l'Agence des coopération de régulateurs de l'énergie (ACER) créée par un règlement $\mathrm{n}^{\circ} 713 / 2009 \mathrm{du}$ Parlement et du Conseil instituant une agence de coopération de régulateurs de l'énergie responsable de la surveillance du respect des codes de réseau européens par les réseaux européens des gestionnaires de réseau de transport pour l'électricité et le gaz; de la même manière, s'agissant du secteur audiovisuel, le CSA participe au réseau de l'ERGA (European Regulators Group for Audiovisual Media Services). Dans un arrêt remarqué, la Cour de justice semble attribuer aux autorités de régulation créées par le droit communautaire le monopole de la définition de l'intérêt communautaire dans les secteurs qui leur sont attribués: Commission européenne c. République fédérale d'Allemagne, Affaire C-424/07, [2009] Rec. C.E. I11431; Aude Bouveresse, "Vers un statut communautaire des régulateurs nationaux? ", (2010) 2 Europe; sur le même sujet : Jacques ZILLER, "Les autorités administratives indépendantes : entre droit interne et droit communautaire ", (2010) R.F.D.A. 901.

99. Commission européenne c. République fédérale d'Allemagne, préc., note 98.

100. Sur ces questions : Aude RouYERE, "Avantages et limites de l'orthodoxie juridique : le cas exemplaire du contentieux des antennes-relais ", dans Mélanges en l'honneur de Pierre Bon, Paris, Dalloz, 2014, p. 1099-1125. 
dit, ces "réseaux " transnationaux produisent des énoncés, qui ne sont pas toujours normatifs en eux-mêmes, mais qui sont interprétés comme tels par les juges suprêmes nationaux dans un souci de cohérence. De fait, il n'est pas étonnant de constater, au nom de l'uniformité, que le pouvoir de police générale au niveau local cède, dans certaines hypothèses, face à l'exercice du pouvoir de police spéciale ${ }^{101}$. La montée en puissance d'un tel phénomène et des nouvelles pratiques administratives qu'il engendre ne peut que retenir l'attention.

S'il convient de suivre Jacques Caillosse lorsqu'il impute la diffusion d'une culture d'évaluation ou l'avènement d'une surdétermination économique du service public à un "libéralisme" ou, plus exactement "au néolibéralisme ", l'argument mériterait d'être affiné. Comme l'a déjà montré Foucault ${ }^{102}$, le néolibéralisme, entendu comme technique de gouvernement, se décline en plusieurs formes ou "instruments "103. La représentation la plus commune de cette domination néolibérale est celle qui voit dans le "marché " un modèle qui exerce son attraction sur les procédés de l'action publique. On dira par exemple, pour en revenir à la surdétermination économique du service public, qu'en faisant primer l'efficacité - ou l'efficience, c'est selon - sur l'égalité, la jurisprudence du Conseil d'État devient de plus en plus perméable

101. Cons. d'Ét. Ass., 26 octobre 2011, Commune de Saint-Denis, commune des Pennes-Mirabeau, A.J.D.A. 2011.2219. Sur la littérature relative à la montée en puissance des phénomènes de "réseaux" de certaines autorités administratives: François LAFARGE, "Administrative Cooperation between Member States and Implementation of EU Law ", (2010) 16 European Public Law 597, 597-616; Irène BELliER, "Unie dans la diversité : la culture administrative de l'Union européenne à la croisée des chemins ", dans Pascal MBongo (dir.), Le phénomène bureaucratique européen, Bruxelles, Bruylant, 2009, aux pages 67-93.

102. Michel Foucault, Naissance de la biopolitique: Cours au Collège de France (1978-1979), coll. "Hautes études ", Paris, Gallimard/Seuil, 2004, qui inspire fortement les travaux de Jacques Caillosse.

103. Pierre Lascoumes et Patrick Le Gales, Gouverner par les instruments, Paris, Presses de Sciences Po, 2004. 
aux impératifs du marché ${ }^{104}$. Une analyse plus poussée des causes de l'envahissement d'une telle surdétermination économique s'impose. Elle passe par un examen attentif des contraintes propres à cette "rationalité économique " triomphante. Les arrêts Million et Marais, Société EDA marquent certes un tournant. Ils ne sont pas les seuls pour autant. En effet, on aurait pu penser que l'intégration du droit de la concurrence dans le bloc de légalité administrative aurait donné naissance à deux courants jurisprudentiels : l'un cherchant à tempérer le droit de concurrence aux nécessités d'intérêt général; l'autre plus rigoureux, se voulant une transposition pure et simple des exigences du droit communautaire de la concurrence. Il semble, contrairement aux intentions affirmées alors par le Conseil d'État105, que le second courant soit en train de s'imposer lentement, mais sûrement. Au-delà du droit des concentrations ${ }^{106}$, l'interprétation du Conseil d'État rejoint désormais à plusieurs égards celle du droit de 1'Union ${ }^{107}$. Ce choix ne doit pas être déconnecté d'un certain consensus politique sur les bienfaits du marché et de la libre concurrence ${ }^{108}$. Telle est, en substance, notre première critique. Si l'on rejoint globalement l'auteur dans ses analyses, nous pensons qu'un examen plus affuté du déplacement

104. Cons. d’Ét. 25 février 2015, n³75724, A.J.D.A. 2015. 421.

105. CONSEIL D'ÉTAT, Rapport public 2002. Collectivités publiques et concurrence, Paris, La documentation française, 2003.

106. Cons. d'Ét. 5 novembre 2014, Société Wienerberger, Rec. Cons. d'Ét., $\mathrm{n}^{\circ} 373065$.

107. Cons. d’Ét. Ord., 5 février 2014, SAS Allocab, n³74524, A.J.D.A. 2014. 661 (étude M. Lombard, S. Nicinski et E. Glaser et Avis de l'Autorité de la concurrence, $\mathrm{n}^{\circ} 13-\mathrm{A}-23$ du 16 décembre 2013 concernant un projet de décret relatif à la réservation préalable des voitures de tourisme avec chauffeur). La solution a été en partie suivie par le Conseil constitutionnel : Cons. const. 17 octobre 2014, VTC, A.J.D.A. 2015. 226, note A. Haquet. Dans le sens d'une convergence globale : Cons. d'Ét. Ass., 30 décembre 2014, Société Armor SNC, R.F.D.A. 2015. 57, concl. B. Dacosta; Azienda Ospedaliero-Universitaria di Careggi-Firenze c. Data Medical Services, Affaire C-568/13, 18 décembre 2014, pts. 33, 34, 35 et 44.

108. Jacques ATTALI (prés.), Rapport pour la libération de la croissance économique française, Paris, La documentation française, 2008, p. 156160 . 
des évolutions de la "rationalité économique " permettrait de mieux en prendre la mesure.

De récentes hypothèses théoriques pourraient servir à mieux identifier ce qui se joue derrière l'emploi des néologismes " marchandisation", " marchéisation" ou "surdétermination économique " du droit. On pense ici aux travaux de Béatrice Hibou $^{109}$, laquelle, revisitant les thèses de Weber, propose de lier la technicité grandissante du droit au développement du néolibéralisme plutôt que de dissocier le marché de l'État. L'originalité de ses travaux tient dans l'idée que le phénomène bureaucratique se développe aussi dans l'entreprise - celle transnationale. Car la représentation d'un marché dont les principaux acteurs seraient l'entrepreneur ou l'entreprise occulte précisément une autre bureaucratie concurrente, celle de l'entreprise. Dès lors, et contrairement à ce qu'une lecture rapide de l'ouvrage de Jacques Caillosse pourrait suggérer, il n'y a pas lieu d'opposer le "marché " à l'État. C'est, au contraire, en comparant le rapprochement des deux types de bureaucratie, celle de l'État et celle de l'entreprise, que l'on peut cerner au plus près ce qui se joue dans la "surdétermination économique " du service public ou la diffusion d'une culture d'évaluation au sein de l'administration. Aussi, l'examen de la recomposition administrative des entreprises transnationales sous l'influence du modèle administratif de la finance s'avère-t-il plus que nécessaire ${ }^{110}$. Une telle lecture prête davantage attention aux évolutions qui imposent une certaine standardisation des normes managériales communes aux secteurs public ou privé. Cette emprise du management privé, sur laquelle plusieurs auteurs ont attiré l'attention ${ }^{111}$, se caractérise par une technique de

109. Béatrice HiBou, La bureaucratisation du monde à l'ère néolibérale, Paris, La découverte, 2012.

110. Alexandra OuRoussoff, Triple A. Une anthropologie dans les agences de notation, coll. "Anthropolis ", Paris, Belin, 2013 (trad. A. Blanchard).

111. Anne PEZET et Eric PEZET, La société managériale: essai sur les nanotechnologies de l'économique et du social, Paris, La ville qui brûle, 2010. 
gouvernement indolore. C'est là, selon nous, qu'il conviendrait de concentrer les efforts d'une approche compréhensive ${ }^{112}$ sur les transformations de l'action publique, au risque sinon de se tromper de cible en pointant l'influence grandissante de "l'économie "113 du droit sur le droit administratif. On se demande même, à bien y réfléchir, si la critique de la "surdétermination économique " du service public liée à l'influence de l'analyse économique du droit ne ferait pas la part belle aux thèses de ses thuriféraires compte tenu de sa trop grande généralité.

La seconde critique est d'ordre méthodologique. D'inspiration sociologique, la position de Jacques Caillosse se rapproche à notre sens de celle développée par le mouvement " critique du droit "114. Or, si cette approche entreprend à juste titre de dévoiler les enjeux politiques sous-jacents au discours des juristes, elle donne parfois des causes très générales à des phénomènes très complexes. Personne ne nie que le droit administratif soit à l'image d'un grand théâtre ${ }^{115}$ où se jouent les transformations de l'État. Ce qui apparaît plus contestable, en revanche, est la corrélation existant entre les transformations du droit administratif et celles de l'État. L'analyse de l'auteur semble partir du principe que les structures qu'elle dévoile n’appartiennent " (...) pas à la conscience mais au comportement, et l'individu n'en acquiert qu'une connaissance restreinte par des prises de consciences incomplètes (...) "116. Or, prendre au sérieux les apports épistémologiques de la philosophie analytique et, plus largement, d'une certaine critique libertarienne des sciences

112. Au sens wébérien du terme.

113. Eric PEZET et Juliette SEnEChAL, Normes juridiques et normes managériales, coll. " Droit et société ", Paris, L.G.D.J./Lextenso, 2014.

114. Xavier DuPRE DE BOUloIs et Martine KAluszynski (dir.), "Droit administratif et critique du droit ", dans Le droit en révolution(s). Regards sur la critique du droit des années 1970 à nos jours, coll. "Droit et société ", vol. 23, Paris, L.G.D.J./Lextenso, 2011, aux pages 85-100.

115. Jacques CAILlosse, "Le droit administratif, ce grand théâtre... ", dans Mathieu DOAT et Gilles DARCY (dir.), L'imaginaire en droit, coll. "Penser le droit ", Bruxelles, Bruylant, 2011, aux pages 260-290.

116. Jean Piaget, Le structuralisme, Paris, P.U.F., 1968, p. 88. 
sociales ${ }^{117}$ incline à inverser la perspective. L'analyse se déploie dès lors vers une approche plus resserrée, permettant de mieux appréhender les causes plurielles de la complexité des phénomènes analysés. Une telle modestie n'a d'autre intérêt que de permettre d'affiner l'analyse des propositions formulées en sciences sociales.

Partant, n'aurait-il pas été plus judicieux que l'auteur circonscrive son objet de manière plus resserrée, ce qui lui aurait permis de mieux établir le lien entre l'exposé des thématiques qu'il aborde et les conclusions qu'il en tire? Autrement dit, la démonstration de Jacques Caillosse n'aurait, à notre sens, rien perdu de sa pertinence si elle avait pris le soin d'identifier plus précisément les contraintes qui pèsent sur les évolutions contemporaines du droit administratif. Or, parce qu'il ne prend pas toujours la peine d'entrer dans le détail de la technique qu'il prétend critiquer, l'auteur prend le risque d'éluder parfois son discours-objet ${ }^{118}$. Un exemple propre à notre champ de recherche : l'introduction d'un référentiel comptable ayant pour modèle celui de la comptabilité d'exercice constitue-t-elle un signe de la privatisation de l'État? Il serait hâtif de tirer une telle conclusion sans s'assurer auparavant de l'usage et des enjeux que présente l'outil comptable pour les principaux acteurs concernés ${ }^{119}$. Ceux-ci peuvent se cantonner, dans des cas ponctuels, à une recherche de valorisation $\mathrm{du}$ patrimoine des collectivités territoriales ou de l'État dans un contexte de raréfaction des deniers publics;

117. Friedrich HAYEK, Scientisme et sciences sociales: essai sur le mauvais usage de la raison, coll. "Agora", Paris, Plon, 1986 (trad. R. Barre); Friedrich HAYEK, Studies in Philosophy, Politics and Economics, Chicago, University of Chicago Press, 1967; Paul K. FEYERABEnd, Knowledge, Science and Relativism, par J. PRESTON (dir.), New York, Cambridge University Press, 1999.

118. Par discours-objet, on se réfère autant à celui des normes qu'à celui de la doctrine: Raymond Boudon, L'idéologie ou l'origine des idées reçues, Paris, Fayard, 1986, p.106-128.

119. Décret $n^{\circ} 2014-524$ du 22 mai 2014 portant modification des règles relatives à l'organisation et au fonctionnement de Pôle emploi, J.O. 24 mai 2014. 
différemment, on peut assister à une utilisation de la comptabilité d'exercice dans un but de légitimation de la privatisation de certaines activités de l'État ${ }^{120}$. Ces quelques illustrations ne sont que des exemples qui, en raison de la complexité dans laquelle ils s'insèrent, permettent de formuler des propositions empiriquement utiles.

Entamer ce débat, ce n'est nullement faire outrage aux travaux de Jacques Caillosse. Au contraire, c'est tirer pleinement les conséquences d'une démarche dont il a été l'un des précurseurs et qu'il convient de réhabiliter à sa juste valeur. Là réside tout l'intérêt d'une science administrative certes plurielle, mais réunie autour d'un socle méthodologique commun. Le coût d'entrée n'en sera pas négligeable à la fois pour les juristes, les politistes ou les sociologues. C'est ce prix qu'il convient pourtant de payer si l'on souhaite réconcilier une science administrative de moins en moins audible par des juristes universitaires toujours plus spécialisés avec les sciences sociales. Réciproquement, la critique adressée aux juristes par les sciences sociales ne peut s'avérer pertinente qu'à la condition qu'elle prenne au sérieux cette même technique juridique qu'elle entend dénoncer ${ }^{121}$.

120. Corine EyRAUD, Le capitalisme au cœur de l'État. Comptabilité privée et action publique, coll. "Dynamiques socio-économiques ", Paris, Du croquant, 2013.

121. C'est d'ailleurs un point important que Jacques Caillosse souligne dans sa préface à l'ouvrage de Patrice Duran : Patrick DuRAn, Penser l'action publique, coll. " Droit et société ", Paris, L.G.D.J. 2010, p. 3-16. 